\title{
Meta
}

Journal des traducteurs

Translators' Journal

\section{Antoine, J. et M.-C. Capiau-Huart (2006) : Dictionnaire des marchés financiers, Bruxelles, De Boeck et Larcier, 687 p.}

\section{Bernard Thiry}

Volume 53, numéro 3, septembre 2008

URI : https://id.erudit.org/iderudit/019253ar

DOI : https://doi.org/10.7202/019253ar

Aller au sommaire du numéro

Éditeur(s)

Les Presses de l'Université de Montréal

ISSN

0026-0452 (imprimé)

1492-1421 (numérique)

Découvrir la revue

Citer ce compte rendu

Thiry, B. (2008). Compte rendu de [Antoine, J. et M.-C. Capiau-Huart (2006) :

Dictionnaire des marchés financiers, Bruxelles, De Boeck et Larcier, 687 p.]

Meta, 53(3), 711-714. https://doi.org/10.7202/019253ar

Ce document est protégé par la loi sur le droit d'auteur. L'utilisation des services d'Érudit (y compris la reproduction) est assujettie à sa politique d'utilisation que vous pouvez consulter en ligne.

https://apropos.erudit.org/fr/usagers/politique-dutilisation/
Cet article est diffusé et préservé par Érudit.

Érudit est un consortium interuniversitaire sans but lucratif composé de l’Université de Montréal, l'Université Laval et l'Université du Québec à Montréal. Il a pour mission la promotion et la valorisation de la recherche. https://www.erudit.org/fr/ 
L'uniformatisation du processus et des procédés de traduction, commandée par les volumes énormes, les critères de qualité et les gains de production, sera ainsi compensée par la nécessaire adaptation des textes aux exigences de chaque client.

Fruit de nombreuses études et enquêtes menées et mises à jour au cours des années 1999 à 2006, ce livre trace un tableau réaliste (certains diront sombre) de la transformation qu'a subie la profession de traducteur au cours de la dernière décennie et de son évolution prévisible à court et à moyen terme. L'intégration de la traduction au sein des «industries de la langue» n'est pas qu'un artifice de langage: elle décrit une profession passée en moins de dix ans du stade artisanal au stade technologique.

Ceux qui songent à entrer dans la profession se poseront sans doute après lecture de ce livre une foule de questions auxquelles il serait bon d'avoir réponse avant d'accéder au marché du travail; ceux qui s'y trouvent déjà y découvriront de nombreuses pistes de réflexion sur la façon de survivre dans un monde où globalisation, informatisation et spécialisation deviennent les clés essentielles du succès.

Serge Marcoux

Université de Montréal, Montréal, Canada

\section{NOTES}

1. Selon les statistiques, le chiffre d'affaires global de la traduction commerciale se serait situé en 2005 entre 9 et 19 milliards de dollars US.

2. Également connu comme DG94/R2T disponible en français à l'adresse www.qualitrad.net.

Antoine, J. et M.-C. Capiau-Huart (2006): Dictionnaire des marchés financiers, Bruxelles, De Boeck et Larcier, 687 p.

Maîtriser un savoir, c'est en maîtriser aussi le nom des concepts discrets. À ce titre, comme M. Jourdain, nous faisons tous de la terminologie sans le savoir. Encore convient-il de se donner les outils experts pour en diffuser le message. Dans l'ouvrage ici recensé coïncident précisément l'érudition de la matière enseignée (les marchés financiers) et la rigueur de l'exposé terminologique. À la vérité, ses auteurs rassemblent amplement les qualités requises pour cet exercice: actifs dans la recherche terminologique et dans l'enseignement des domaines traités, il n'en sont pas, de surcroît, à leur premier essai puisqu'un autre ouvrage sur le sujet, et qui a déjà connu deux éditions, figure dans la même collection: Titres et Bourse (Vol. 1: Valeurs mobilières, Vol. 2: Marchés, $2^{\mathrm{e}}$ éd., $2^{\mathrm{e}}$ tirage, 1999, 445 et 502 p.).

Dans la production dictionnairique des Langues de spécialité, voici donc une somme, remarquable tant par le volume de son contenant que par la qualité de son contenu; un dictionnaire dont a besoin notre époque, au moment où les savoirs explosent, où, par «les mots du savoir», le transfert des connaissances s'impose comme une nécessité et où, par la langue, qui est le vecteur privilégié du savoir, l'accès aux domaines de spécialité devient un enjeu d'éducation et de démocratie.

Quantitativement, les 2231 entrées de ce dictionnaire, centré sur le strict domaine des marchés financiers, attestent la profondeur de l'analyse, dans un domaine qui se complexifie sans cesse par l'internationalisation, l'abondance de l'information et la sophistication des techniques et des instruments financiers.

Les entrées (mot, syntagme ou sigle) sont associées à un numéro, qui permet d'établir un rapport aisé avec les index des équivalents dans pas moins de cinq autres langues: anglais, allemand, espagnol, italien et néerlandais.

À ce titre, ce dictionnaire est, du reste, généreusement multilingue - caractère que partagent également de nombreux ouvrages inclus dans la riche bibliographie. La plupart 
des entrées sont, en effet, assorties d'une liste d'équivalents dans ces cinq langues citées. Certes, ces «traductions» (parler d'équivalent est méthodologiquement plus approprié: on ne traduit pas, on recherche l'équivalent notionnel attesté dans le système de l'autre langue) sont réduites au minimum nécessaire et leur simple mention semble laisser entendre que les langues citées et les systèmes par lesquels celles-ci traduisent le domaine sont tout à fait isomorphes. C'est loin d'être toujours aussi simple, bien entendu - et les auteurs n'en sont point dupes quand ils glosent, en ce sens, les termes qui s'imposent en anglais (voyez, par exemple, les cas de polysémies signalées pour les anglicismes: Benchmarking, Spin-off ou Strip, v.gr. Voyez aussi le soin de leur recherche, tel qu'il est révélé, par exemple encore, par les nombreux synonymes espagnols donnés pour équivalents de Dividende privilégié). On conçoit toutefois aisément que le «service» de proposer ces équivalents ne pouvait se doubler d'une longue étude monographique supplémentaire dans chacune des autres langues mentionnées.

Parmi les autres rubriques dont se compose chaque article, certaines ont un caractère linguistique (relatif à la dénomination), les autres, un caractère encyclopédique (relatif à la notion et à son référent).

Les entrées sont immédiatement suivies d'un renvoi à des synonymes et antonymes (V. Grand actionnaire), puis d'une définition, attentive aux nombreux problèmes de polysémie (Coentreprise, Gestion de patrimoine, Obligation, Titrisation) et illustrée d'exemples et d'autres données d'explication encyclopédique: schémas et graphiques (Échelle de note, Tableau d'amortissement) et formules mathématiques (Duration, Moyenne mobile).

Définitions et exemples sont, eux-mêmes, sources d'un intéressant système de renvois à des corrélats. Avec une autre rubrique "Ne pas confondre avec», c'est ainsi à tout le réseau hautement structuré des notions du domaine et de leurs relations que, à la manière d'un hypertexte, ce dictionnaire donne accès. Le lecteur dispose, de la sorte, d'un outil d'approfondissement par approches successives des notions qui constituent le domaine.

L'étymologie offre un complément linguistique et de contenu de bon aloi pour la compréhension de certains termes (ex.: Soulte et Mezzanine ou Blanchiment de capitaux et Scriptophilie). Les notes d'érudition historique (Bourse, Krach boursier) vont dans le même sens.

Par ailleurs, des marques de variétés diatopiques introduisent judicieusement la distinction des langues et des systèmes nationaux qu'elles recouvrent. Voir BSA (FR), Branche 21 (BE), Perte de cours (CH), Capital émis (CA), Shark Watcher (US), Stag (GB).

De sorte que ce dictionnaire est tout à la fois explicatif, encyclopédique ("analytique» disent les auteurs) et traductif. Il constitue, en effet, tour à tour, une base de données terminologiques (le relevé des termes du domaine), une base de connaissance - et non seulement de données - (les notions structurées du domaine et leurs relations, suggérées par des renvois aux corrélats) et une base d'équivalences multilingues.

Autre chose encore aurait été d'en faire une base de données discursives, en y ajoutant les aspects phraséologiques, à savoir la manière dont les termes s'emploient dans le concret du discours (conditions syntagmatiques d'emploi, collocations et cooccurrences, etc.), soit tout ce qui permet non seulement la compréhension du domaine, assurée à suffisance ici (décodage), mais aussi la production langagière adéquate (encodage).

Parmi les outils de base dont s'est dotée la lexico-terminographie, les marques grammaticales auraient pu également trouver ici un place avantageuse. Il y va, ici aussi, de l'assistance à l'encodage correct de la langue de spécialité en question (un Espagnol dit «una orden» et «el banco»; il s'exposerait à un sourire amusé en appliquant, par ignorance, le même genre grammatical à l'équivalent français de ces termes). Ainsi, sans l'indication de son genre grammatical, le non-spécialiste est incapable de maîtriser l'emploi en discours du terme stochastique, p. ex. Ainsi aussi, pour les anglicismes, dit-on un ou une sin shares, strip ou swap? Reconnaissons que, dans la plupart des cas, l'exemple - dont c'est aussi le 
rôle, du reste - compense utilement l'information (Voyez «La courbe représentant le stochastique...» et "La réalisation d'un swap...»). N'empêche, la mention du genre (indécis dans l'usage quelquefois) pourrait donner lieu à un débat intéressant et à une proposition de solution argumentée. Ainsi, le cas de Holding est-il résolu, dans les exemples, en faveur du féminin (selon la logique de l'abréviation de «une société holding»), mais le dictionnaire reste silencieux sur les raisons du doute qui fait que d'autres autorités se prononcent en faveur du double genre et certaines même en faveur du seul masculin.

En ce qui concerne l'aspect notionnel, à présent, non contents de nous guider de main de maître par ce qu'il faut bien appeler le désordre alphabétique, à travers cette énorme richesse en vrac, voici que les auteurs nous offrent, de ce domaine - microcosme de l'économie -, une vision organique d'une cohérence étonnamment synthétique, réussissant à réduire à huit le nombre de domaines thématiques essentiels autour desquels gravite toute notion inhérente aux marchés financiers. Et l'élégance diamantaire de l'octogone qui illustre cette synthèse (voir couverture et p. 11) n'a d'égale que sa lumineuse efficacité pour la compréhension des mécanismes expliqués. "Ainsi, l'acquisition (opération) - sur la base d'une analyse préalable - sur le marché financier, par un investisseur (acteur), d'un instrument financier, constitue pour ledit investisseur, un placement dont il espère une performance, mais pour lequel il accepte d'assumer un certain risque» (p. 10).

À caractère thématique également, et complémentaires de l'ordre alphabétique du dictionnaire, les schémas conceptuels constituent une autre qualité remarquable de ce dictionnaire. Leur grande clarté contribue aux objectifs didactiques et cognitifs qui sont l'enjeu de cet ouvrage. Ces schémas, dynamiques, sont fondés sur des critères notionnels de distinction genre-espèce; lus de gauche à droite, ils développent une méthodologie résolument onomasiologique, partant de la notion pour rendre compte des dénominations. Betty Cohen (Lexique de cooccurrents: Bourse, Montréal, Linguatech, 1986) avait donné, de la méthode et sur le même domaine de la Bourse, un modèle remarquable, aboutissant à un classement extrêmement complet des cooccurrents des termes de la Bourse. Le modèle est fécond et mérite d'être imité.

L'ouvrage fait montre, par ailleurs, d'un effort louable de francisation dans un domaine largement dominé par l'anglais. Ainsi, Coentreprise a droit à l'entrée principale (à laquelle renvoie l'entrée secondaire Joint Venture). De plus, sous les entrées anglaises qui s'imposent même dans l'usage francophone, figurent, dans l'immense majorité des cas, leurs équivalents français (Private banking, Family office semblent faire exception), sous la rubrique «Syn.» (Performance globale sous Return) ou sous la rubrique «f.» (Jeune pousse sous Startup, Plan d'affaires sous Business plan ou Options de souscription d'actions sous Stock option), soit encore sous les deux rubriques (pour les nombreux équivalents proposés de Junk bond, p. ex.), soit enfin en ample note explicative ("Barattage», "Moulinage» sous Churning).

La sitographie (p. 687) témoigne de la consultation judicieuse de la banque de données terminologiques du "Service de la langue française» du ministère de la Communauté française de Belgique. La consultation des listes que publie régulièrement, dans le Journal Officiel, la Délégation générale à la langue française et aux langues de France (http://www. culture.gouv.fr/culture/dglf) aurait permis, par ailleurs, de combler quelques silences (Benchmarking: référenciation, étalonnage, parangonage) ou de compléter les mentions de néologismes reconnus (Business plan: plan d'affaires, mais aussi Plan de développement).

Le dictionnaire s'adresse aux professionnels de la finance, professeurs, formateurs et étudiants en finance; aux investisseurs individuels et au grand public, «auquel (il) procure les clés indispensables pour une meilleure compréhension du monde financier»; aux traducteurs et interprètes financiers et économiques. Bref, un ouvrage expert, à l'érudition solide, et non seulement dans les moindres recoins du sujet, mais aussi sur les données d'histoire ou d'étymologie utile (voir ci-dessus). Ouvrage expert donc, et sur une langue de spécialité qui l'est tout autant - même si elle n'est pas exempte d'humour, avec ses «zinzins» 
(les investisseurs institutionnels), ses joyaux de la couronne et ses bijoux de famille, ou son Dentiste belge et sa Veuve de Carpentras!), le tout dans une terminologie, par ailleurs, largement imagée (voyez l'interprétation des graphes en papillon, condor et mouette ou encore en arbre de Noël).

L'ouvrage a été salué avec enthousiasme par la critique: pour Le Monde (20/06/06), «après la bible de la Finance d'entreprise [...], cet ouvrage pourrait devenir son équivalent pour les marchés financiers», tandis que Bruno Colmant (L'Écho, 20/03/06) y voit «une source de référence pour les praticiens» et lui reconnaît «un intérêt indéniable pour ceux qui recherchent une analyse systématique du langage de la finance» (Fucam Liaisons, n ${ }^{\circ} 44$, février 2007, p. 14). Nous joignons sans réserves notre modeste part à ces éloges mérités.

Bernard Thiry

Université de Liège, Bruxelles, Belgique

\section{Miao, J. (2006): On the Development of Translation Competence Through Translation Instruction, Tianjin, Tianjin People's Publishing House, $\mathrm{x}+193 \mathrm{p}$.}

More and more translators/interpreters are needed in various fields in our global village, which has led to an emphasis on translation pedagogy to produce eligible, qualified translators/interpreters to meet the needs of an era of globalization and cross-cultural communication. In retrospect, as we examine the teaching of translation in past decades, an emerging problem is conspicuous - the lack of a consistent, systematic and scientific methodology/pedagogy in translation teaching. Translation teaching is featured by anecdotal reflections and fragmentary experience. Therefore, scholars of Translation Studies have come to realize that we need to identify theoretical hypothesis based on actual teaching practice and to construct general principles, rules, and methods that govern or underlie such practice. On the Development of Translation Competence Through Translation Instruction tries to meet this need, and intends to devise a pedagogical model of translation instruction based on consistent, systematic, and appropriate concepts, principles, and the methods that will lay a foundation for translation instruction, and is intended as a contribution to the profession that is in need of such enhancement.

The book comprises five chapters: Introduction, Research on Translation Competence, Empirical Study on Translation Competence, A Model of Translation Instruction, and Conclusion. Its introduction presents an outline that highlights the major points and emphasis of the intended research. The second chapter "Research on Translation Competence" establishes the basis of the contents leading to a logical development of the research. As translation is both a purposeful human activity and a complex mental activity, the translator's performance in fulfilling the tasks requires a unique competence involving a comprehensive application of various knowledge and expertise. It has been a comprehensive competence that has not been adequately described and clearly defined and the development of this unique competence has not been systematically investigated. Translation competence is comprised of interrelated sub-competences and components involved in the translation process. In defining translation competence, the present author intends to analyze translation competence mainly from the cognitive, linguistic and communicative perspectives with the belief that it encompasses three categories contributed by different components. The classification of the three categories manifests a more systematic, comprehensive, hierarchical, and interrelated view on translation competence as a whole. It reflects both a macroscopic examination of the holistic translation competence and a microscopic analysis of the discrete components. In addition, the present author postulates that translation competence is a professional mental competence to deal with complex 